\title{
Dot Symbol Auto-filling Method for Land-use Areas in Cartographic Representation
}

\author{
Yong Yin ${ }^{\mathrm{a},}$ *, Chengcheng Zhang ${ }^{\mathrm{a}}$ \\ ${ }^{a}$ Chinese Academy of Surveying and Mapping, Beijing 100830, China, yinyong@casm.ac.cn; Chengcheng Zhang, \\ 94100594@qq.com \\ * Corresponding author
}

Keywords: Complex areas, Dot symbol auto-filling, Delaunay triangulation, Fine division, Boundary constraint

\begin{abstract}
:
Dot symbol filling is a key step in the visualization of land use areas data. The traditional method cannot adapt well to the shape features of complex areas, which leads to the unreasonable symbol arrangement in the inner region and boundary of areas during symbol filling. For this reason, a dot symbol auto-filling method for land-use areas considering shape features is proposed in this paper. Firstly, based on the constrained Delaunay triangulation, the internal structure of complex areas is branched and the paragraphs with the same width and narrowness features in the branch are extracted to realize the fine division of complex areas. Then, according to the geometric shape features of the segment, it is divided into three types, i.e., tile type, narrow type and point type, filled with three levels of symbols: point, line and plane respectively. Finally, the internal and external buffer constraints are set to process the symbols at the boundary of areas to optimize the filling effect of boundary symbols. Based on the national data of a region in Guizhou Province, the method in this paper is compared with the traditional symbol filling methods for validation. The experimental results show that the proposed method has better filling fullness for symbol filling of complex areas, and the symbols at the boundary fit areas contour without creating spatial conflicts such as cover, and the filling result can better reflect the shape features of the areas.
\end{abstract}

